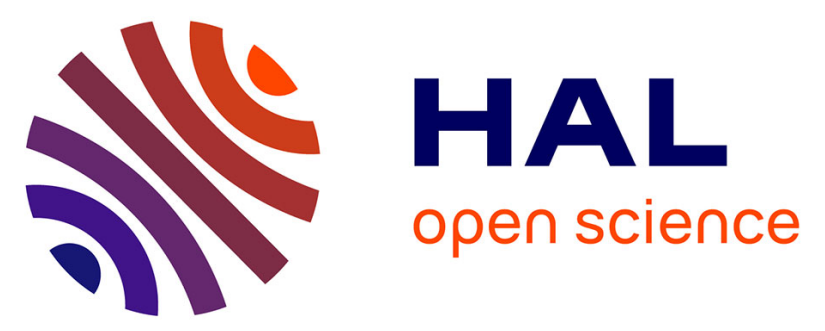

\title{
Relationships between brain metabolism decrease in normal aging and changes in structural and functional connectivity.
}

Gaël Chételat, Brigitte Landeau, Eric Salmon, Igor Yakushev, Mohamed Ali Bahri, Florence Mézenge, Audrey Perrotin, Christine Bastin, Alain Manrique, Armin Scheurich, et al.

\section{To cite this version:}

Gaël Chételat, Brigitte Landeau, Eric Salmon, Igor Yakushev, Mohamed Ali Bahri, et al.. Relationships between brain metabolism decrease in normal aging and changes in structural and functional connectivity.. NeuroImage, 2013, 76, pp.167-77. 10.1016/j.neuroimage.2013.03.009 . inserm-00806846

\section{HAL Id: inserm-00806846 https://www.hal.inserm.fr/inserm-00806846}

Submitted on 2 Apr 2013

HAL is a multi-disciplinary open access archive for the deposit and dissemination of scientific research documents, whether they are published or not. The documents may come from teaching and research institutions in France or abroad, or from public or private research centers.
L'archive ouverte pluridisciplinaire HAL, est destinée au dépôt et à la diffusion de documents scientifiques de niveau recherche, publiés ou non, émanant des établissements d'enseignement et de recherche français ou étrangers, des laboratoires publics ou privés. 
Relationships between brain metabolism decrease in normal aging and changes in structural and functional connectivity

Gaël Chételat ${ }^{1,2,3,4^{*}}$, Brigitte Landeau ${ }^{1,2,3,4^{*}}$, Eric Salmon ${ }^{5}$, Igor Yakushev ${ }^{6,7}$, Mohamed Ali Bahri $^{5}$, Florence Mézenge $e^{1,2,3,4}$, Audrey Perrotin ${ }^{1,2,3,4}$, Christine Bastin $^{5}$, Alain Manrique ${ }^{8}$, Armin Scheurich ${ }^{6}$, Mathias Scheckenberger ${ }^{9}$, Béatrice Desgranges ${ }^{1,2,3,4}$, Francis Eustache $^{1,2,3,4}$, Andreas Fellgiebel $^{7}$

${ }^{1}$ INSERM, U1077, Caen, France

${ }^{2}$ Université de Caen Basse-Normandie, UMR-S1077, Caen, France

${ }^{3}$ Ecole Pratique des Hautes Etudes, UMR-S1077, Caen, France

${ }^{4}$ CHU de Caen, U1077, Caen, France

${ }^{5}$ Cyclotron Research Centre, University of Liège, Allée du 6 Août, 8, B30, 4000 Liège, Belgium

${ }^{6}$ Department of Psychiatry and Psychotherapy, University Medical Centre Mainz, Mainz, Germany

${ }^{7}$ Department of Nuclear Medicine, Technische Universität München, Ismaninger Str. 22, 81675 Munich, Germany

${ }^{8}$ Department of Nuclear Medicine, CHU de Caen, Caen, France

${ }^{9}$ Department of Nuclear Medicine, University Medical Center Mainz, Mainz, Germany

\section{${ }^{*}$ Equally contributed}

Corresponding author: Dr Gaël Chételat, Unité de Recherche U1077, Centre Cyceron, Bd H. Becquerel, BP 5229, 14074 Caen Cedex, France.

e-mail: chetelat@ cyceron.fr; Tel: +33 (0)2 314701 73; Fax: +33 (0)2 31470275 


\begin{abstract}
Normal aging is characterized by brain glucose metabolism decline predominantly in the prefrontal cortex. The goal of the present study was to assess whether this change was associated with age-related alteration of white matter (WM) structural integrity and/or functional connectivity. FDG-PET data from 40 young and 57 elderly healthy participants from two research centres $(n=49 / 48$ in Centre 1/2) were analyzed. WM volume from T1weighted MRI (Centre 1), fractional anisotropy from diffusion-tensor imaging (Centre 2), and resting-state fMRI data (Centre 1) were also obtained. Group comparisons were performed within each imaging modality. Then, positive correlations were assessed, within the elderly, between metabolism in the most affected region and the other neuroimaging modalities. Metabolism decline in the elderly predominated in the left inferior frontal junction (LIFJ). LIFJ hypometabolism was significantly associated with macrostructural and microstructural WM disturbances in long association fronto-temporo-occipital fibers, while no relationship was found with functional connectivity. The findings offer new perspectives to understand normal aging processes and open avenues for future studies to explore causality between agerelated metabolism and connectivity changes.
\end{abstract}

Keywords: normal aging, connectivity, diffusion tensor imaging, resting-state fMRI, FDGPET metabolism, frontal cortex.

Abbreviations: DTI, diffusion tensor imaging; FA, fractional anisotropy; GM, grey matter LIFJ, left inferior frontal junction; PVE, partial volume effects; SPM, Statistical Parametric Mapping; VBM, voxel-based morphometry; WM, white matter. 


\section{Introduction}

In vivo cross-sectional ${ }^{18}$ FDG-PET studies in normal healthy individuals have consistently shown decreased cerebral glucose metabolism with age, with a differential effect across brain regions, the frontal cortex showing the greatest effects (Moeller et al., 1996; Petit-Taboué et al., 1998; Garraux et al., 1999; Herholz et al., 2002; Zuendorf et al., 2003; Kalpouzos et al., 2009; Hsieh et al., 2012). The most frequently reported areas are the ventral and dorsal lateral prefrontal / inferior frontal cortex, the anterior cingulate cortex, medial prefrontal areas and precentral and perisylvian areas. Decreased metabolism is also reported, though less consistently, in the caudate nuclei and the (superior) lateral temporal cortex. The neurophysiological mechanisms underlying these metabolic changes with age are still unknown. It is possible however that connectivity disruption is involved.

Indeed, normal aging process is also characterized by changes within the WM. In addition to WM hyperintensities (e.g. Maillard et al., 2012), structural and functional connectivity changes occur and alterations of WM tract integrity have been consistently reported. Thus, histopathological studies have shown age-related loss of myelinated fibers and degeneration of myelin in the WM (Peters, 2002; Marner et al., 2003). Diffusion tensor imaging (DTI) allows in-vivo investigation of WM microstructure integrity (Le Bihan, 2003) with fractional anisotropy (FA) being the best established DTI indices for the quantification of structural integrity and connectivity. DTI studies in normal aging have reported significant decrease in FA in the whole brain (Rovaris et al., 2003), with greater FA decline in frontal compared to posterior WM regions (Pfefferbaum et al., 2000, 2005; Abe et al., 2002; Salat et al., 2005; Ota et al., 2006; Sullivan et al., 2006). In general, an anterior-posterior gradient is found, for example with the genu of the corpus callosum being more significantly altered than the splenium (Pfefferbaum et al., 2000; Sullivan et al., 2001; Kochunov et al., 2007, 2012). Also, highest rates of decline are reported for the late thinly myelinated associative tracts, while the 
thickly myelinated motor and sensory tracts are more resistant to normal aging processes (Tang et al., 1997; Bartzokis et al., 2001, 2003, 2004; Kochunov et al., 2007), giving rise to the developmental theory. In addition to microstructure changes, macroscopic changes in WM structural integrity can also be assessed using T1-weighted MRI and measures of WM volume for example using voxel-based morphometry (VBM; Good et al., 2001). Thus, voxelwise WM volume decrease with age has been reported in the frontal lobe, optic radiations, and posterior limb of internal capsule (Good et al., 2001). Finally, in addition to these WM structural changes, resting-state fMRI studies in normal aging consistently reported functional connectivity disruption with age specifically along the anterior-posterior axis of the brain (Andrews-Hanna et al., 2007; Meunier et al., 2009; Biswal et al., 2010; Grady et al., 2010; Jones et al., 2011; Wu et al., 2011; Mevel et al., 2012), though they mainly focused on midline structures of the default mode network (Raichle et al., 2001).

To further understand the processes underlying age-related brain changes, it seems of particular interest to assess whether there is any relationship between cerebral metabolism decline with age, and alterations in structural and functional WM connection tracts. It is likely that glucose metabolism may impact on myelin integrity as reflected by FA measurements as maintaining myelin is energy demanding; conversely, myelin disruption is expected to impact on metabolism and functioning of connected brain regions (Bartzokis, 2004). The density and volume of WM fibers, that probably influences both FA and VBM measures of WM volume, are also very likely to impact on the projection sites; it has been shown for example in Alzheimer's disease that the profile of hypometabolism is at least partly due to disconnection processes, i.e., disruption of WM tracts that project to these metabolically affected regions (Villain et al., 2008, 2010a). Finally, local metabolic dysfunction may be due to, or may lead to, disruption in the functional connectivity with distant brain regions. 
Although showing a correlation between two processes does not allow to infer causality between these processes, assessing the relationships between brain metabolism in regions typically affected by normal aging on the one hand, and structural and functional connectivity on the other hand, is a first step to further our understanding of the physiological mechanisms underlying age-related metabolic decline. A few studies assessed the links between microstructural WM changes and metabolism (Kochunov et al., 2009; Inoue et al., 2008 ; Kuczynski et al., 2010), but there has been no study to date that used a multimodal approach to assess whether metabolism decrease with age is related to microscopic or macroscopic structural or functional connectivity changes.

The objective of the present study was thus to assess the relationships between age-related grey matter (GM) decrease of glucose metabolism measured with FDG-PET, focusing on the region with the strongest effect, and macrostructural (with VBM-WM volume), microstructural (with DTI), and functional (with resting-state fMRI) connectivity voxelwise throughout the whole brain.

\section{Material and methods}

\subsection{Participants}

The study included 97 healthy participants separated in young ( $<40$ years old) and elderly (>60 years old) individuals pooled across two research centres (Centre $1=$ Caen in France, and Centre 2 = Mainz in Germany) in the framework of a tripartite collaborative project that already gave rise to a publication (Bastin et al., 2012). Participants showed normal performances for age in a battery of neuropsychological tests, had no clinical evidence of psychiatric or neurological disorders, no severe brain lesion on T2-weighted or FLAIR MRI images, were free of medication that could affect cognitive functioning, and reported being in 
good health. All participants gave informed consent to cognitive and neuroimaging assessments, which were approved by the local ethics committee in each centre.

The number of participants per group and centre and their corresponding demography are provided in Table 1. All participants from both centres had an FDG-PET scan. Structural T1MRI images from Centre 1 were used to assess WM volume as a reflect of WM macroscopic structural integrity. DTI-FA data were available in all individuals from Centre 2, allowing to assess WM microscopic structural integrity. Finally, resting-state fMRI data were available from all individuals from Centre 1, allowing to measure functional connectivity.

\subsection{Neuroimaging data acquisition}

\subsubsection{FDG-PET data}

In both centres, PET data were acquired under standard resting conditions after participants had fasted for at least 6h. In Centre 1, a 10-min PET scan was acquired on a Discovery RX VCT 64 PET/CT device (General Electric Healthcare) about 50 min after intravenous injection of $180 \mathrm{MBq}$ FDG. The device has a resolution of 3.76 × 3.76 x $4.9 \mathrm{~mm}$ (field of view $=157 \mathrm{~mm}$ ). Images were reconstructed with a resultant voxel size of $2.7 \times 2.7 \times 3.27$ mm. In Centre 2, a 15-min PET scan was acquired in list mode on a Philips Gemini TF PET/CT scanner (Philips Medical Systems, Eindhoven, NL) about 30 min after intravenous injection of approximately $150 \mathrm{MBq}$ FDG. The PET camera has an axial field of view of 18 $\mathrm{cm}$ and an axial resolution of $4.7 \mathrm{~mm}$. Images corrected for scatter and attenuation were reconstructed to $5 \mathrm{~mm}$ slices. In each centre, brain metabolic activity was measured during quiet wakefulness with eyes closed and ears unplugged. 


\subsubsection{MRI data}

MRI was performed within three months of the PET exam. Subjects were equipped with earplugs and their heads were stabilized with foam pads to minimize head motion. In Centre 1, images were acquired on a Philips (Eindhoven, The Netherlands) Achieva 3T scanner. In Centre 2, data acquisition was performed on a Siemens Trio 3T scanner.

\subsubsection{T1-weighted structural MRI}

A T1-weighted anatomical image was obtained in all participants of both Centres. In Centre 1, high-resolution T1-weighted images were obtained using the 3D fast field echo sequence (3DT1-FFE sagittal; repetition time $=20 \mathrm{~ms}$, echo time $=4.6 \mathrm{~ms}, 170$ slices; slice thickness 1 $\mathrm{mm}$, field of view $=256 \mathrm{~mm}$, matrix size 256x256). In Centre 2, acquisition was performed with the following parameters: repetition time $=1170 \mathrm{~ms}$, echo time $=2.38 \mathrm{~ms}, 244$ slices, slice thickness $0.82 \mathrm{~mm}$, field of view $=210 \mathrm{~mm}$, matrix size $256 \times 256$.

\subsubsection{DTI data}

In Centre 2, diffusion-weighted images were also obtained in all young and elderly participants. Images were obtained using a diffusion-weighted single-shot spin-echo echoplanar based sequence ( 30 directions; $b=1000 \mathrm{~s} / \mathrm{mm}^{2}$; matrix 128x128; section thickness, $3 \mathrm{~mm}$; voxel size, $1.5 \times 1.5 \times 3 \mathrm{~mm}$; repetition time $=7100 \mathrm{~ms}$, echo time $=102 \mathrm{~ms}$ ).

\subsubsection{Resting-state fMRI data}

In Centre 1, resting-state fMRI time series were obtained in all young and elderly participants using an interleaved 2D T2*SENSE (SENSitivity Encoding) echo-planar imaging sequence $(2 \mathrm{D}-\mathrm{T} 2 *$-FFE-EPI axial, $\mathrm{SENSE}$ factor $=2$; repetition time $=2382 \mathrm{~ms}$; echo time $=30 \mathrm{~ms}$; flip angle $=80 \circ ; 42$ slices; slice thickness $=2.8 \mathrm{~mm}$; no gap; in-plane resolution $=2.8 \times 2.8$ 
$\mathrm{mm}^{2} ; 280$ volumes). During the acquisition, subjects were asked to relax, lie still in the scanner and keep their eyes closed while not falling asleep. The first six volumes were discarded due to saturation effects.

\subsection{Image preprocessing and analyses}

A schematic overview of all preprocessing steps for all imaging modalities is provided in Figure 1. In brief, all images from a same individual were coregistered together and spatially normalized using the same normalization parameters. These parameters were obtained from the corresponding coregistered structural T1-MRI, that was itself segmented and spatially normalized to the MNI stereotactic space using the VBM5.1 toolbox (http://dbm.neuro.uni-jena.de), implemented in the Statistical Parametric Mapping (SPM) 5 software (Wellcome Trust Centre for Neuroimaging, London, UK). Modality-specific steps were then performed as described below and in the Figure 1, and all images were smoothed and masked before being included in the statistical analyses.

\subsubsection{FDG-PET data preprocessing}

For FDG-PET data, a "double-scaling" method was applied to erase the effects of centre (see Supplementary Figure 1): first, in order to control for individual variation in global FDG uptake, each normalized PET image was divided by the values extracted from a region of interest in the pons. Then the resulting individual PET images were divided, voxelwise, by the mean of all PET images from the corresponding centre to account for inter-centre effects. Note that applying the second scaling allowed to annihilate any main effect of centre while group effects remained unchanged (data not shown). Images were then masked to include only voxels with values higher than $80 \%$ of the mean of each centre (PET-mask), then 
smoothed and masked (see below for smoothing and masking steps common to all imaging modalities).

PET data were not corrected for partial volume effects (PVE) in the main analyses of this article. This is usually the case in multi-centre studies because PVE-correction relies on the GM segments issued from T1-MRI images so that it may induce an additional bias in PET images due to inter-centre differences in the quality of MR images and resulting segments. In the present study, the GM-WM contrast of T1-MRI images and consequently the GM segments were very different between both centres so that the PVE-correction would have introduced a significant bias in the analyses. However, complementary analyses were conducted to ensure that the results were not merely a reflect of atrophy (see below Complementary analyses in the Result section).

\subsubsection{Structural MRI data preprocessing: WM volume maps}

The WM segments issued from the structural MRI data using the VBM5.1 routine were modulated to correct for non-linear warping so that values in resultant images were expressed as volume corrected for brain size. Resultant images were smoothed and masked (see below). As explained above for PVE correction and GM segments, the segmentation of T1-MRI images allowing to obtain WM segments depends on the GM-WM contrast of T1-MRI images. As the contrast differed between centres, WM images from Centre 1 and 2 could not be pooled together for these analyses. Because images from Centre 1 showed greater GMWM contrast, only images from Centre 1 were used in the main analyses of the article. Complementary analyses were however conducted on WM images from Centre 2 to allow a direct comparison between DTI and WM findings (see below Complementary analyses in the Result section). 


\subsubsection{DTI data preprocessing}

Diffusion-weighted images were corrected for subject motion and eddy currents using FSL 4.1 (FMRIB Analysis Group, Oxford, UK, http://www.fmrib.ox.ac.uk/fsl). Gradients were adjusted accordingly by applying the rotational part of the resulting affine transformations (Tournier et al., 2011). Nonbrain voxels were removed using FSL-BET (Smith, 2002). In order to calculate FA maps, a single diffusion tensor was fitted to the data using the fiber tractography toolkit CAMINO v. 2 (Microstructural Imaging Group, University College London, UK, http://web4.cs.ucl.ac.uk/research/medic/camino/pmwiki/pmwiki.php). FA images were then coregistered on the T1-MRI and spatially normalized using the normalization parameters obtained through the VBM5.1 routine from the corresponding T1MRI. Resulting images were smoothed and masked (see below).

\subsubsection{Resting-state fMRI data preprocessing and first-level analyses}

Processing of resting-state fMRI data was adapted from Mevel et al. (2012) where the method is described and illustrated in more details. Briefly, fMRI time series were corrected for slice timing, realigned to the mean functional image using rigid body transformations, warped to a non-EPI T2* scan to reduce geometrical distortions effects (Villain et al., 2010b), spatially normalized with the T1-MRI parameters and smoothed with a 4mm FWHM kernel. A bandpass filter $(0.01-0.08 \mathrm{~Hz})$ was then applied to the images using the AFNI toolbox and the resulting images were masked with a binary "GM-MRI-mask" created from the segmented mean GM T1 volume in conjunction with the mean non EPI-T2* volume in the MNI space (including only voxels with values above 0.25 in both mean images; see Villain et al., 2010b; Mevel et al., 2012). The resulting images were entered in the first-level fMRI data analyses. As we were interested in assessing the functional connectivity of the peak area of hypometabolism with age, the most significant peak of the comparison analysis of FDG-PET 
images between young and elderly individuals was used as the centre coordinates of the seedof-interest for the functional connectivity analyses. A $6 \mathrm{~mm}$ radius spherical seed centred on these coordinates was thus created using the SPM MarsBar toolbox and the mean time-course in this seed-of-interest was extracted for each of the 49 individuals. Time course was also extracted in the whole brain as well as in the WM and cerebrospinal fluid using ROIs corresponding to the voxels with the highest value in the mean WM and cerebrospinal fluid images obtained from the VBM processing of T1-MRI images. These data were extracted to be used as nuisance variables in the first-level analyses of fMRI datasets.

To obtain individual functional connectivity maps, first-level functional connectivity analyses were performed. For each individual, a regression analysis was performed with the timecourse in the seed-of-interest as the independent variable, the preprocessed fMRI time series as the dependent variable, and the six movement parameters generated from realignment, as well as the global, WM and cerebrospinal fluid time courses and their derivatives as nuisance covariates. The resulting individual SPM-T maps were converted to z-maps applying a Fisher's z transform, and the corresponding individual functional connectivity z-maps were smoothed and masked (see below) before being entered in second-level group analyses.

\subsubsection{Differential smoothing}

Since images had different original spatial resolutions according to the imaging centre and the imaging modalities, and also taking into account the intermediate smoothing in the preprocessing of fMRI data, a differential smoothing has been applied to each imaging modality to equalize their effective smoothness (Chételat et al., 2008, 2010; Villain et al., 2008; Villain et al., 2010a). Thus, Gaussian kernels of 9.3x9.3x 8.7 (x y z) $\mathrm{mm}^{3}$ for FDG-PET data from Centre 1, of $8.8 \mathrm{~mm}$ for FDG-PET data from Centre 2, of $10 \mathrm{~mm}$ for structural MRI WM data from Centre 1 , of $8.7 \mathrm{~mm}$ for resting-state fMRI data from Centre 1 and of 
9.9x9.9x $9.5 \mathrm{~mm}^{3}$ for DTI-FA data from Centre 2 were applied to obtain a final resolution of $10 \mathrm{~mm}$ for all images of any centre and modality, based on the following formula: original resolution ${ }^{2}+1^{\text {st }} \operatorname{smooth}^{2}\left(+2^{\text {nd }}\right.$ smooth $\left.^{2}\right)=$ final smooth ${ }^{2}$ (Poline et al., 1995; Chételat et al., 2008).

\subsubsection{Final masking}

All images from any modality should be masked using the same GM or WM mask so that all analyses on GM or WM are performed on the same voxels and can thus be compared. A conjunction was thus performed between the PET-mask (taking into account images from both centres) and the GM MRI-mask (taking into account both the structural MRI images and the non EPI-T2* images). All FDG-PET and RS-fMRI connectivity preprocessed and smoothed images were then masked using this final GM conjunction mask. Similarly, all DTIFA and WM volume preprocessed and smoothed images were masked using a same final WM mask corresponding to the conjunction of the WM masks from both centers. These were obtained using the centre means of GM and WM segments and including only voxels having greater probability of being WM than GM. The resulting masked images were entered in the following statistical group analyses.

\subsection{Statistical group analyses}

The aim of this study was to assess whether there was any relationship between metabolic changes with age, and age-related changes in structural and functional connectivity. Thus, for each modality, two kinds of analyses were performed: group comparisons between young and elderly, and correlations, within the elderly, between metabolic changes in the region of most significant difference and each other modality. 
For FDG-PET data, images from both centres ( $\mathrm{n}=21$ young and 28 elderly in Centre1 and $\mathrm{n}=19$ young and 29 elderly in Centre 2) were first entered in an ANOVA with two factors (group and centre) of two levels each and controlling for gender and education. Betweengroup differences (young minus elderly contrast) were assessed within each centre, and a conjunction analysis was then performed on the contrast from both centres to highlight the regions showing an effect of group independent from the centre (Salmon et al., 2003). Also, the coordinates of the peak of greatest between-group difference from this analysis were used to determine the centre of a $6 \mathrm{~mm}$ spherical seed-of-interest as described above for the resting-state fMRI functional connectivity analysis. This seed-of-interest was also used for the regression analysis between age-related hypometabolism and the other imaging modalities. Thus, the mean FDG-PET metabolism in this seed was extracted for each individual and used as a covariate in the regression analyses described below.

Group comparison analyses were then performed for each other modality to assess differences between young and elderly individuals. Analyses on WM volume were conducted on data from Center 1 ( $\mathrm{n}=21$ young and 28 elderly), analyses on DTI-FA were conducted on the 19 young and 29 elderly participants from Center 2, and analyses on LIFJ connectivity were conducted on 21 young and 28 elderly participants from Center 1.

Note that for WM volume analyses, the volume of the cerebrospinal fluid obtained from the VBM routine was also entered as a nuisance variable to avoid boundary effects (i.e. effects in the boundary between the cerebrospinal fluid and the WM due to ventricular dilatation). Note also that for resting-state fMRI connectivity, one-sample analyses were first performed within the young and within the elderly to illustrate the functional connectivity of each group. Then, group comparisons were performed as for the other modalities.

Finally, regression analyses were performed between metabolism in the seed-of interest and the other modalities in regions showing a significant effect of age (using an inclusive mask 
from the corresponding analysis). Thus, for WM structural MRI data for example, correlations were assessed within regions showing significant differences in the previous comparison of WM volume between young and elderly. Regression analyses were performed within the elderly using data from Center $1(n=28)$ for WM volume, data from Center $2(n=29)$ for DTIFA, and data from Center $1(n=28)$ for LIFJ connectivity.

Our objectives being to assess whether age-related metabolic decrease was associated with connectivity disruption, comparisons and regressions were only assessed in the expected directions (elderly < young and positive correlations). Gender and years of education were systematically controlled for in all analyses described above. Results were usually displayed with different thresholds: i) a more stringent $\mathrm{p}$ (FDR-corrected) threshold $<0.05$ with number of contiguous voxels $\mathrm{k}>20$; note that a still more severe threshold was used if needed, i.e. if differences were significant in the whole $\mathrm{GM}$ or $\mathrm{WM}$ at $\mathrm{p}(\mathrm{FDR})<0.05$; and ii) a more liberal threshold of $\mathrm{p}$ (uncorrected) 0.005 with $\mathrm{k}>50$, to avoid false negative and highlight less significant but still probably meaningful findings. Localisation in the GM (FDG-PET and functional connectivity from resting-state fMRI) was based on the atlas of the human brain from Duvernoy and Bourgouin (1999) and localisation in the WM (VBM-WM and DTI-FA) was based on the MRI atlas of the human WM from Oishi et al. (2010).

\section{Results}

\subsection{Group comparisons}

\subsubsection{FDG-PET}

The conjunction analysis highlighting differences between young and elderly common to both centres showed highly significant decrease metabolism in the elderly compared to the young in the entire brain GM at $\mathrm{p}_{\mathrm{FDR}}<0.05$, and even at $\mathrm{p}_{\mathrm{Fwe}}<0.05$ (see Supplementary Figure 2A), so that results were displayed at $\mathrm{p}_{\mathrm{Fwe}}<10^{-5}$ to illustrate brain areas of most significant changes 
(Figure 2). The most significant peak (MNI coordinates x y z: -56 12 28) was located in the left inferior frontal junction (LIFJ) close to the pars triangularis of the inferior frontal gyrus (BA44) in a cluster encroaching both the ventrolateral and dorsolateral prefrontal cortex and the anterior premotor cortex. The seed-of-interest obtained from this peak (see above) is illustrated in Figure 2. Similar differences (in terms of significance and extend) were found in the right hemisphere counterpart. The regions of most significant differences also include the insula, medial dorsal and ventral frontal areas and anterior cingulate cortex, angular gyrus and thalamus.

\subsubsection{WM volume}

Significant differences in WM volume predominated in two clusters, an anterior (frontal) one and a more posterior (parietal) one, none of them surviving the p FWE-corrected threshold (Supplementary Figure 2B and Figure 3). The frontal cluster involved the frontal corpus callosum (genu), the uncinate fasciculus, and the anterior part of the inferior fronto-occipital fasciculus. The posterior cluster included the central and parieto-occipital part of the corpus callosum (body and splenium), the posterior part of the inferior fronto-occipital fasciculus, the inferior longitudinal fasciculus, and the posterior part of the cingulum bundle.

\subsubsection{DTI}

Group comparison of FA maps revealed highly significant decrease in FA in the elderly compared to the young in the entire brain $\mathrm{WM}$ at $\mathrm{p}_{\mathrm{FDR}}<0.05$ (Supplementary Figure 2C). Areas of most significant differences shown at $\mathrm{p}_{\mathrm{FWE}}<0.05$ included most frontal WM fibers (particularly the anterior part -the genu - of the corpus callosum), the fornix, the superior and inferior longitudinal fasciculus, the inferior fronto-occipital fasciculus, the rostral part of the cingulum, and the uncinate fasciculus (Figure 3). In total, the profile of WM changes was 
very similar to that observed using T1-MRI WM volume maps though more significant, with an extensive overlap between both modalities when displayed at different thresholds (see Figure 3).

\subsubsection{Functional connectivity}

Figure 4 illustrates the results of the one-sample analyses of functional connectivity with the LIFJ for each group. In young participants, the functional connectivity of the LIFJ concerned the whole lateral frontal cortex (also extending to superior parietal areas including the lateral part of the post-central gyrus and the angular gyrus), posterior part of the middle and inferior temporal gyrus (encroaching the fusiform gyrus), anterior superior temporal gyrus, insula, and a small portion of the dorsomedial frontal cortex. In old participants the profile was similar with the exception of the temporal regions (superior, middle and inferior temporal cortex, insula and fusiform gyrus), so that connectivity included the same frontal lateral areas extending to superior parietal regions as well as a small portion of the dorsomedial frontal cortex. The profiles of LIFJ functional connectivity in both young and old participants were globally bilateral with left predominance.

The direct comparison of LIFJ functional connectivity between young and elderly revealed higher connectivity in the young participants in the insula and anterior superior and middle posterior temporal cortex, as well as a small cluster straddling both the post-central and the inferior parietal gyri (Figure 4 and Supplementary Figure 2D).

\subsection{Regression analyses with LIFJ metabolism}

To assess whether age-related changes in metabolism were related to structural or functional connectivity changes, regression analyses were performed between metabolism in the region of greatest decrease with age (the LIFJ), and WM volume, FA, and LIFJ functional 
connectivity in the elderly. As mentioned above, regressions were restricted to brain areas showing a significant effect of age in the respective modality, using an explicit mask obtained from the previous analyses of group comparison and applying a statistical threshold adapted to the modality (see Supplementary Figure 2 caption). This strategy was preferred to avoid exploring the whole brain in one modality and no voxel in another modality with a fixed threshold (for example with a $\mathrm{p}(\mathrm{FDR})>0.05$ for all modalities), but results were very similar and the conclusion remained unchanged when using different thresholds for the mask.

\subsubsection{LIFJ metabolism versus WM volume}

LIFJ metabolism significantly correlated to WM volume in the inferior longitudinal fasciculus and the anterior part of the corpus callosum body (Figure 5).

\subsubsection{LIFJ metabolism versus FA}

Significant correlations were found between LIFJ metabolism and FA in the uncinate fasciculus, the inferior longitudinal fasciculus (both surviving the FDR-corrected threshold), the rostral part of the cingulum - frontal portion of the corpus callosum, and the superior longitudinal fasciculus (Figure 6).

\subsubsection{LIFJ metabolism versus functional connectivity}

There was no region where LIFJ metabolism correlated with LIFJ functional connectivity.

For the sake of clarity, all the results described in details above are summed up in Figure 7. 


\subsection{Complementary analyses}

Because of significant differences in the GM/WM contrast of T1-MRI images between both Centres, very significant Centre effects were found in the analyses of both GM and WM volume data (data not shown). For this reason and as mentioned above, only T1-MRI images from Centre 1 were included in WM volume VBM analyses, and FDG-PET images were not corrected for PVE.

However, complementary analyses were performed to ensure that 1) LIFJ hypometabolism was not merely the reflect of GM atrophy; and 2) the discrepancy between age effects on WM volume versus DTI-FA was not due to the fact that these analyses were conducted on different samples. Thus, the group comparison analysis of FDG-PET data was repeated but only including data from Centre 1 that were first corrected for PVE (PMOD Technologies Ltd., Adliswil, Switzerland). This analysis allowed to show that, using PVE-corrected FDG-PET data, LIFJ hypometabolism was recovered at the same stringent $\mathrm{p}$ (FWE- corrected) $<10^{-5}$ threshold (Supplementary Figure 3), so that it is not merely a reflect of atrophy. Second, although the segmentation clearly differed from that obtained from Centre 1 images, a separate group comparison analysis was performed using the WM volume data from Centre 2 for the sake of comparison with the group comparison analysis of DTI-FA data. The results are shown in Supplementary Figure 4 and they showed that, even when assessed from the same samples, DTI-FA showed more significant and extended age-effects compared to WM volume.

Finally, although it was not the objective of the present study, we also computed complementary analyses to provide basic information about the relationships between LIFJ hypometabolism and cognitive performances. Regression analyses were thus performed, within the elderly, between metabolism in the LIFJ seed and cognitive performances from the neuropsychological battery, selecting tests that were common between both centres and 
including years of education as a covariate. We found a significant positive correlation with working memory (forward and backward digit span; $\mathrm{p}=0.004$ and 0.03 respectively) and executive functions (Trail Making Test Part B; $\mathrm{p}=0.03$ - a trend was found for B-A; $\mathrm{p}=$ 0.053) but not with episodic memory (immediate and delayed recall and recognition; all $\mathrm{p}$ values > 0.1) tasks (data not shown). Using a global cortical FDG-PET measure as covariate to assess the specificity of the relationship, only the relationship with the forward digit span remains significant.

\section{Discussion}

Thanks to a multimodal approach, the present study aimed at giving a comprehensive overview of the links between age-related metabolism decrease measured with FDG-PET and changes in microstructural (DTI), macrostructural (WM volume) integrity and functional connectivity. It showed that predominant frontal, and especially LIFJ, metabolic decrease with age correlated with structural but not with functional connectivity changes. More specifically significant relationships were found with both microstructural and macrostructural WM integrity in long association fronto-temporo-occipital fibers.

This study also offers a comprehensive overview of the effects of age on different imaging modalities. The findings highlight massive metabolism decrease in elderly compared to young participants in the lateral and medial frontal cortex, together with significant though less marked changes in posterior temporo-parietal and middle and superior temporal cortex. Elderly were also characterized by structural alterations of WM fibers predominating in the frontal lobe but also involving parietal and temporal fibers, with more significance for microstructural DTI-FA than for macrostructural volumetric changes, and by functional connectivity disruption between the LIFJ and the superior temporal and inferior parietal 
cortex. Although this study was not specifically designed to test for regional differences in the effects of age, the most significant differences between young and elderly were observed in the frontal lobe for both metabolism and FA, suggesting a predominant effect of age in this region. This would be in line with a large amount of previous studies that have consistently demonstrated that the anterior / frontal part of the brain is the most sensitive to normal aging processes (see Introduction). The main hypothesis to explain the pattern of brain changes with age is the "last-in-first-out" or developmental theory (Grieve et al., 2005) according to which the regions that are the most sensitive to age effects are the last ones to emerge phylogenetically and ontogenetically. This applies to both metabolic changes (Kalpouzos et al., 2009) and WM deterioration where age effects predominate in associative tracts that maturated later and are thinly myelinated (Davis et al., 2009; Giorgio et al., 2010; Bartzokis et al., 2012; Kochunov et al., 2012). Two additional general comments arose from the frontal predominance of age-related changes. First, it is interesting to further highlight the contrast with the pattern found in Alzheimer's disease where alterations predominate in the temporal and parietal lobes, in brain structures of the default mode network (Buckner et al., 2008). Second, this major involvement of the frontal lobe in normal aging agrees with the neuropsychological profile of healthy elders characterized by predominant executive deficits, given the well-known role of the prefrontal cortex in executive functions (Raz and Rodrigue, 2006, for a review). More specifically, the LIFJ region highlighted in the present study is part of an attention / frontoparietal control / executive network (Seeley et al., 2007) and is known to be critically involved in executive functions or cognitive control including working memory, task switching and inhibitory control (Brass et al., 2005; Derrfuss et al., 2012; Schroeter et al., 2012; Sundermann and Pfleiderer, 2012). That LIFJ metabolism correlated with working memory and, to a lesser degree, executive function in the present study supports 
this view, though further confirmatory analyses would be needed notably to assess how specific this relation is.

The patterns of age-associated changes also highlight similarities and differences between WM changes depicted using DTI-FA versus VBM-WM volume. On the first hand, it is notable that both analyses showed consistent and clearly overlapping profiles despite the differences in the techniques and the fact that analyses were conducted on two independent subsamples. On the other hand, the effects were much more marked for FA than for volume decrease and this was also found when WM volume analyses were conducted on the same sample (see Complementary analyses in the Results section). This is consistent with previous reports showing that DTI-based measures are more sensitive than T1-derived WM volume to detect age-related changes in WM integrity (Fjell et al., 2008; Giorgio et al., 2010). This may reflect an increase sensitivity of the method, or the fact that age has a more dramatic effect on the microstructure than on the macrostructure of the WM. Both hypotheses are not exclusive, and they probably both contribute to the predominance of FA changes; DTI is expected to be more sensitive to T1-weighted MRI-based WM volume measures on the one hand, and microstructural changes may occur before and/or without macrostructural manifestation (Salat et al., 2005; Vernooij et al., 2009; Westlye et al., 2010) while the reverse is unlikely on the other hand.

The profile of functional connectivity of the LIFJ is similar to that described in Sundermann and Pfleiderer (2012; Figure 1) assessing IFJ co-activations or resting-state fMRI connectivity, and consistent with the well known fronto-parietal control network (Spreng and Schacter, 2012; see also above). In healthy elders, LIFJ connectivity was reduced in the insula, posterior part of the superior temporal cortex and inferior parietal cortex. There is no previous study assessing the effect of age on the IFJ connectivity as most previous resting- 
state fMRI studies focused on the default mode network (reporting an anterior-posterior disruption within this network; see Andrews-Hanna et al., 2007, for instance) or used different approaches or seeds so that the findings are hardly comparable. However, our findings suggest a disruption of the fronto-temporal and, to a less extend fronto-parietal communication, which is consistent with the pattern of structural connectivity alteration involving frontal, parietal and temporal fibers in the present study using DTI or VBM.

The main question in this study was whether metabolism decline with age in the LIFJ, as the main peak of FDG-PET dysfunction in normal elders, correlated with structural or functional connectivity. We found that LIFL hypometabolism with age correlated with age-related structural disconnection, and these relationships mainly concern long association fibers connecting the frontal to the temporal and occipital lobes (uncinate and longitudinal fasciculus), or frontal to parietal regions (rostral cingulum) or both frontal lobes together (frontal corpus callosum). By contrast, no significant relationship was found between agerelated changes in LIFL metabolism and functional connectivity. As far as we know there have only been three studies assessing the link between FDG-PET and WM tract changes. Inoue et al. (2008) correlated FA in the genu and splenium of the corpus callosum and metabolism throughout the whole brain (i.e. the reverse compared to our analysis) and found a significant relationship with FA in the genu only, and metabolism in the prefrontal cortex. Although direct comparisons between this study and ours are made difficult due to differences in the approaches (especially the use of different seeds), the results are nonetheless very close as we also found the corpus callosum genu FA to correlate with LIFJ metabolism. Kochunov et al. (2009) reported strong correlations between FA and FDG in the WM and especially in the corpus callosum. In GM cortical areas, only age-corrected correlations were reported and, though all positive (with $\mathrm{r}$ values ranging from 0.2 to 0.4 ), they were not statistically 
significant. However, the relationships were strong and significant before age-correction (personal communication), which is thus consistent with our findings. Finally, Kuczynski et al. (2010) correlated FA in specific WM regions of interest and FDG-PET metabolism in 16 individuals ranging from normal to demented. Although the population differed compared to ours, they reported a significant relationship between FA in the left temporal WM - a region recovered in both our VBM-WM and DTI analyses, and metabolism in a frontal region strikingly similar to our LIFJ seed. They concluded that the major WM tracts that seem to be associated with metabolism are the longitudinal fasciculus and those associated with frontotemporal connectivity, both tracts also found to be the most associated to LIFJ metabolism decline in the present study together with the anterior corpus callosum.

In total, although there are only few and methodologically different studies to compare with, findings are overall consistent. They indicate that the predominant LIFJ metabolism decline in normal aging, probably in part responsible for age-related cognitive control decline, is associated with structural disruption not only in frontal but also in long distant associative WM tracts. As mentioned in the Introduction, it is possible that the loss or degeneration of myelinated fibers decreases synaptic activity in surrounding or projecting cells and/or that decrease metabolism results in WM alterations because it alters myelinisation in proximate or projecting tracts or because it reflects degeneration in neuronal cell bodies itself resulting in the degeneration of associated axons. Again, showing a correlation does not indicate a causal relationship and even more does not indicate the direction of the relation i.e. whether WM disruption is responsible for metabolism decline or the reverse. Future longitudinal studies may help answering this as illustrated in Villain et al. ( 2010a) in Alzheimer's disease.

There are several strengths and limitations in this study. Strengths include the voxelwise multimodal approach allowing to provide a comprehensive view on the relationships between 
metabolism and connectivity changes in normal aging where different aspects of connectivity are considered (microstructural versus macrostructural; structural versus functional). The use of data from two different centres was also an advantage in the present study as it allowed to increase both young and elderly groups sample size and avoid detecting centre or sample specific findings. Moreover, a sophisticated methodology has been designed, where data preprocessing scheme was designed to limit artificial differences across modalities, and where an original scaling method has been used to annul inter-centre effects. By contrast, we decided not to correct PET data for PVE because differences in the quality of the GM segments used for PVE correction between both centres would have induced significant biases in the analyses. As a result, changes in metabolism may partly reflect volume changes. However, complementary analyses conducted on PVE-corrected PET data from a subsample of the participants (Centre 1) showed that LIFJ hypometabolism was still highly significant when correcting for PVE, i.e. a large part of it was not due to atrophy. Another limitation of the present study lay in its cross-sectional design, which may induce cohort effects. Also, as cerebrospinal fluid or PET measures of $\beta$-amyloid abnormalities have not been obtained in our samples, it is possible that the results are partly contaminated by preclinical Alzheimer's disease-related pathological processes (Sperling et al., 2011). Finally, the present study explored the association of major age-related changes in cerebral glucose metabolism with macro- and microstructural surrogate markers as well as with measures of functional connectivity and provides, beyond the specific LIFJ-related findings, a comprehensive multimodal imaging approach. Further studies are needed, however, to complete the picture and provide, using this approach in addition to the detection of vascular and other Alzheimer's disease-related pathologies, a comprehensive answer to the fundamental question of the age-related mechanisms of metabolism decline in association to structural and 
functional connectivity as well as cognitive performance, which is crucial for the understanding of both normal aging and preclinical degenerative diseases. 


\section{Role of the funding source}

The French Caen study was supported by Agence Nationale de la Recherche (ANR

LONGVIE 2007), Programme Hospitalier de Recherche Clinique (PHRC National 2011),

Fondation Plan Alzheimer (Alzheimer Plan 2008 -2012), Région Basse Normandie and

Institut National de la Santé et de la Recherche Médicale (INSERM).

The German study was supported by the Research Focus on Interdisciplinary Neurosciences IFSN of the University of Mainz, Germany.

\section{Acknowledgments}

The authors are grateful to V. de La Sayette, M. Fouquet, N. Villain, R. La Joie, K. Mevel, S.

Egret, C. Schupp, J. Dayan, A. Quillard, L. Barré, A. Abbas, C. Lebouleux, M.H. Noel and M.C. Onfroy for their help in data acquisition.

\section{Disclosure Statement}

There is no conflict of interest for any of the authors. 


\section{References}

Abe, O., Aoki, S., Hayashi, N., Yamada, H., Kunimatsu, A., Mori, H., Yoshikawa, T., Okubo, T., Ohtomo, K., 2002. Normal aging in the central nervous system: quantitative MR diffusion-tensor analysis. Neurobiol. Aging 23, 433-441.

Andrews-Hanna, J.R., Snyder, A.Z., Vincent, J.L., Lustig, C., Head, D., Raichle, M.E., Buckner, R.L., 2007. Disruption of large-scale brain systems in advanced aging. Neuron 56, 924-935.

Bartzokis, G., 2004. Age-related myelin breakdown: a developmental model of cognitive decline and Alzheimer's disease. Neurobiol. Aging 25, 5-18.

Bartzokis, G., Beckson, M., Lu, P.H., Nuechterlein, K.H., Edwards, N., Mintz, J., 2001. Agerelated changes in frontal and temporal lobe volumes in men: a magnetic resonance imaging study. Arch. Gen. Psychiatry 58, 461-465.

Bartzokis, G., Cummings, J.L., Sultzer, D., Henderson, V.W., Nuechterlein, K.H., Mintz, J., 2003. White matter structural integrity in healthy aging adults and patients with Alzheimer disease: a magnetic resonance imaging study. Arch. Neurol. 60, 393-398.

Bartzokis, G., Sultzer, D., Lu, P.H., Nuechterlein, K.H., Mintz, J., Cummings, J.L., 2004. Heterogeneous age-related breakdown of white matter structural integrity: implications for cortical "disconnection” in aging and Alzheimer's disease. Neurobiol. Aging 25, 843-851.

Bartzokis, G., Lu, P.H., Heydari, P., Couvrette, A., Lee, G.J., Kalashyan, G., Freeman, F., Grinstead, J.W., Villablanca, P., Finn, J.P., Mintz, J., Alger, J.R., Altshuler, L.L., 2012. Multimodal Magnetic Resonance Imaging Assessment of White Matter Aging Trajectories Over the Lifespan of Healthy Individuals. Biol. Psychiatry 72, 1026-1034. 
Bastin, C., Yakushev, I., Bahri, M.A., Fellgiebel, A., Eustache, F., Landeau, B., Scheurich, A., Feyers, D., Collette, F., Chételat, G., Salmon, E., 2012. Cognitive reserve impacts on inter-individual variability in resting-state cerebral metabolism in normal aging. Neuroimage 63, 713-722.

Biswal, B.B., Mennes, M., Zuo, X.-N., Gohel, S., Kelly, C., et al., 2010. Toward discovery science of human brain function. Proc. Natl. Acad. Sci. U.S.A. 107, 4734-4739.

Brass, M., Derrfuss, J., Forstmann, B., von Cramon, D.Y., 2005. The role of the inferior frontal junction area in cognitive control. Trends Cogn. Sci. 9, 314-316.

Buckner, R.L., Andrews-Hanna, J.R., Schacter, D.L., 2008. The brain's default network: anatomy, function, and relevance to disease. Ann. N. Y. Acad. Sci. 1124, 1-38.

Chételat, G., Desgranges, B., Landeau, B., Mézenge, F., Poline, J.B., de la Sayette, V., Viader, F., Eustache, F., Baron, J.-C., 2008. Direct voxel-based comparison between grey matter hypometabolism and atrophy in Alzheimer's disease. Brain 131, 60-71.

Chételat, G., Villemagne, V.L., Bourgeat, P., Pike, K.E., Jones, G., Ames, D., Ellis, K.A., Szoeke, C., Martins, R.N., O’Keefe, G.J., Salvado, O., Masters, C.L., Rowe, C.C., Group, the A.R., 2010. Relationship between atrophy and beta-amyloid deposition in Alzheimer's disease. Ann. Neurol. 67, 317-324.

Davis, S.W., Dennis, N.A., Buchler, N.G., White, L.E., Madden, D.J., Cabeza, R., 2009. Assessing the effects of age on long white matter tracts using diffusion tensor tractography. Neuroimage 46, 530-541.

Derrfuss, J., Vogt, V.L., Fiebach, C.J., von Cramon, D.Y., Tittgemeyer, M., 2012. Functional organization of the left inferior precentral sulcus: dissociating the inferior frontal eye field and the inferior frontal junction. Neuroimage 59, 3829-3837.

Duvernoy, H.M., Bourgouin, P., 1999. The Human Brain: Surface, Three-Dimensional Sectional Anatomy With Mri, and Blood Supply. Springer. 
Fjell, A.M., Westlye, L.T., Greve, D.N., Fischl, B., Benner, T., van der Kouwe, A.J.W., Salat, D., Bjørnerud, A., Due-Tønnessen, P., Walhovd, K.B., 2008. The relationship between diffusion tensor imaging and volumetry as measures of white matter properties. Neuroimage 42, 1654-1668.

Garraux, G., Salmon, E., Degueldre, C., Lemaire, C., Laureys, S., Franck, G., 1999. Comparison of impaired subcortico-frontal metabolic networks in normal aging, subcortico-frontal dementia, and cortical frontal dementia. Neuroimage 10, 149-162.

Giorgio, A., Santelli, L., Tomassini, V., Bosnell, R., Smith, S., De Stefano, N., JohansenBerg, H., 2010. Age-related changes in grey and white matter structure throughout adulthood. Neuroimage 51, 943-951.

Good, C.D., Johnsrude, I.S., Ashburner, J., Henson, R.N., Friston, K.J., Frackowiak, R.S., 2001. A voxel-based morphometric study of ageing in 465 normal adult human brains. Neuroimage 14, 21-36.

Grady, C.L., Protzner, A.B., Kovacevic, N., Strother, S.C., Afshin-Pour, B., Wojtowicz, M., Anderson, J.A.E., Churchill, N., McIntosh, A.R., 2010. A multivariate analysis of agerelated differences in default mode and task-positive networks across multiple cognitive domains. Cereb. Cortex 20, 1432-1447.

Grieve, S.M., Clark, C.R., Williams, L.M., Peduto, A.J., Gordon, E., 2005. Preservation of limbic and paralimbic structures in aging. Hum. Brain Mapp. 25, 391-401.

Herholz, K., Salmon, E., Perani, D., Baron, J.C., Holthoff, V., Frölich, L., Schönknecht, P., Ito, K., Mielke, R., Kalbe, E., Zündorf, G., Delbeuck, X., Pelati, O., Anchisi, D., Fazio, F., Kerrouche, N., Desgranges, B., Eustache, F., Beuthien-Baumann, B., Menzel, C., Schröder, J., Kato, T., Arahata, Y., Henze, M., Heiss, W.D., 2002. Discrimination between Alzheimer dementia and controls by automated analysis of multicenter FDG PET. Neuroimage 17, 302-316. 
Hsieh, T.-C., Lin, W.-Y., Ding, H.-J., Sun, S.-S., Wu, Y.-C., Yen, K.-Y., Kao, C.-H., 2012. Sex- and age-related differences in brain FDG metabolism of healthy adults: an SPM analysis. J. Neuroimaging 22, 21-27.

Inoue, K., Ito, H., Uchida, S., Taki, Y., Kinomura, S., Tsuji, I., Sato, S., Horie, K., Kawashima, R., Ito, M., Fukuda, H., 2008. Decrease in glucose metabolism in frontal cortex associated with deterioration of microstructure of corpus callosum measured by diffusion tensor imaging in healthy elderly. Hum. Brain Mapp. 29, 375-384.

Jones, D.T., Machulda, M.M., Vemuri, P., McDade, E.M., Zeng, G., Senjem, M.L., Gunter, J.L., Przybelski, S.A., Avula, R.T., Knopman, D.S., Boeve, B.F., Petersen, R.C., Jack, C.R., Jr, 2011. Age-related changes in the default mode network are more advanced in Alzheimer disease. Neurology 77, 1524-1531.

Kalpouzos, G., Chételat, G., Baron, J.-C., Landeau, B., Mevel, K., Godeau, C., Barré, L., Constans, J.-M., Viader, F., Eustache, F., Desgranges, B., 2009. Voxel-based mapping of brain gray matter volume and glucose metabolism profiles in normal aging. Neurobiol. Aging 30, 112-124.

Kochunov, P., Thompson, P.M., Lancaster, J.L., Bartzokis, G., Smith, S., Coyle, T., Royall, D.R., Laird, A., Fox, P.T., 2007. Relationship between white matter fractional anisotropy and other indices of cerebral health in normal aging: tract-based spatial statistics study of aging. Neuroimage $35,478-487$.

Kochunov, P., Ramage, A.E., Lancaster, J.L., Robin, D.A., Narayana, S., Coyle, T., Royall, D.R., Fox, P., 2009. Loss of cerebral white matter structural integrity tracks the gray matter metabolic decline in normal aging. Neuroimage 45, 17-28.

Kochunov, P., Williamson, D.E., Lancaster, J., Fox, P., Cornell, J., Blangero, J., Glahn, D.C., 2012. Fractional anisotropy of water diffusion in cerebral white matter across the lifespan. Neurobiol. Aging 33, 9-20. 
Kuczynski, B., Targan, E., Madison, C., Weiner, M., Zhang, Y., Reed, B., Chui, H.C., Jagust, W., 2010. White matter integrity and cortical metabolic associations in aging and dementia. Alzheimers Dement. 6, 54-62.

Le Bihan, D., 2003. Looking into the functional architecture of the brain with diffusion MRI. Nat. Rev. Neurosci. 4, 469-480.

Maillard, P., Carmichael, O., Fletcher, E., Reed, B., Mungas, D., DeCarli, C., 2012. Coevolution of white matter hyperintensities and cognition in the elderly. Neurology $79,442-448$.

Marner, L., Nyengaard, J.R., Tang, Y., Pakkenberg, B., 2003. Marked loss of myelinated nerve fibers in the human brain with age. J. Comp. Neurol. 462, 144-152.

Meunier, D., Achard, S., Morcom, A., Bullmore, E., 2009. Age-related changes in modular organization of human brain functional networks. Neuroimage 44, 715-723.

Mevel, K., Landeau, B., Fouquet, M., La Joie, R., Villain, N., Mézenge, F., Perrotin, A., Eustache, F., Desgranges, B., Chételat, G., 2012. Age effect on the default mode network, inner thoughts, and cognitive abilities. Neurobiol. Aging.

Moeller, J.R., Ishikawa, T., Dhawan, V., Spetsieris, P., Mandel, F., Alexander, G.E., Grady, C., Pietrini, P., Eidelberg, D., 1996. The metabolic topography of normal aging. J. Cereb. Blood Flow Metab. 16, 385-398.

Oishi, K., Faria, A.V., Zijl, P.C.M. van, Mori, S., 2010. MRI Atlas of Human White Matter 2nd Edition. Academic Press.

Ota, M., Obata, T., Akine, Y., Ito, H., Ikehira, H., Asada, T., Suhara, T., 2006. Age-related degeneration of corpus callosum measured with diffusion tensor imaging. Neuroimage $31,1445-1452$.

Peters, A., 2002. The effects of normal aging on myelin and nerve fibers: a review. J. Neurocytol. 31, 581-593. 
Petit-Taboué, M.C., Landeau, B., Desson, J.F., Desgranges, B., Baron, J.C., 1998. Effects of healthy aging on the regional cerebral metabolic rate of glucose assessed with statistical parametric mapping. Neuroimage 7, 176-184.

Pfefferbaum, A., Sullivan, E.V., Hedehus, M., Lim, K.O., Adalsteinsson, E., Moseley, M., 2000. Age-related decline in brain white matter anisotropy measured with spatially corrected echo-planar diffusion tensor imaging. Magn. Reson. Med. 44, 259-268.

Pfefferbaum, A., Adalsteinsson, E., Sullivan, E.V., 2005. Frontal circuitry degradation marks healthy adult aging: Evidence from diffusion tensor imaging. Neuroimage 26, 891899.

Poline, J.B., Worsley, K.J., Holmes, A.P., Frackowiak, R.S., Friston, K.J., 1995. Estimating smoothness in statistical parametric maps: variability of p values. J. Comput. Assist. Tomogr. 19, 788-796.

Raichle, M.E., MacLeod, A.M., Snyder, A.Z., Powers, W.J., Gusnard, D.A., Shulman, G.L., 2001. A default mode of brain function. Proc. Natl. Acad. Sci. U.S.A. 98, 676-682.

Raz, N., Rodrigue, K.M., 2006. Differential aging of the brain: patterns, cognitive correlates and modifiers. Neurosci. Biobehav. Rev. 30, 730-748.

Rovaris, M., Iannucci, G., Cercignani, M., Sormani, M.P., De Stefano, N., Gerevini, S., Comi, G., Filippi, M., 2003. Age-related changes in conventional, magnetization transfer, and diffusion-tensor MR imaging findings: study with whole-brain tissue histogram analysis. Radiology 227, 731-738.

Salat, D.H., Tuch, D.S., Greve, D.N., van der Kouwe, A.J.W., Hevelone, N.D., Zaleta, A.K., Rosen, B.R., Fischl, B., Corkin, S., Rosas, H.D., Dale, A.M., 2005. Age-related alterations in white matter microstructure measured by diffusion tensor imaging. Neurobiol. Aging 26, 1215-1227. 
Salmon, E., Garraux, G., Delbeuck, X., Collette, F., Kalbe, E., Zuendorf, G., Perani, D., Fazio, F., Herholz, K., 2003. Predominant ventromedial frontopolar metabolic impairment in frontotemporal dementia. Neuroimage 20, 435-440.

Schroeter, M.L., Vogt, B., Frisch, S., Becker, G., Barthel, H., Mueller, K., Villringer, A., Sabri, O., 2012. Executive deficits are related to the inferior frontal junction in early dementia. Brain 135, 201-215.

Seeley, W.W., Menon, V., Schatzberg, A.F., Keller, J., Glover, G.H., Kenna, H., Reiss, A.L., Greicius, M.D., 2007. Dissociable intrinsic connectivity networks for salience processing and executive control. J. Neurosci. 27, 2349-2356.

Smith, S.M, 2002. Fast robust automated brain extraction. Hum. Brain. Mapp. 17,143-155.

Sperling, R.A., Aisen, P.S., Beckett, L.A., Bennett, D.A., Craft, S., Fagan, A.M., Iwatsubo, T., Jack, C.R., Kaye, J., Montine, T.J., Park, D.C., Reiman, E.M., Rowe, C.C., Siemers, E., Stern, Y., Yaffe, K., Carrillo, M.C., Thies, B., Morrison-Bogorad, M., Wagster, M.V., Phelps, C.H., 2011. Toward defining the preclinical stages of Alzheimer's disease: Recommendations from the National Institute on Aging and the Alzheimer's Association workgroup. Alzheimers Dement 7, 280-292.

Spreng, R.N., Schacter, D.L, 2012. Default network modulation and large-scale network interactivity in healthy young and old adults. Cereb Cortex 22, 2610-2621.

Sullivan, E.V., Rosenbloom, M.J., Desmond, J.E., Pfefferbaum, A., 2001. Sex differences in corpus callosum size: relationship to age and intracranial size. Neurobiol. Aging 22, 603-611.

Sullivan, E.V., Adalsteinsson, E., Pfefferbaum, A., 2006. Selective age-related degradation of anterior callosal fiber bundles quantified in vivo with fiber tracking. Cereb. Cortex 16, $1030-1039$. 
Sundermann, B., Pfleiderer, B., 2012. Functional connectivity profile of the human inferior frontal junction: involvement in a cognitive control network. BMC Neurosci. 13, 119. Tang, Y., Nyengaard, J.R., Pakkenberg, B., Gundersen, H.J., 1997. Age-induced white matter changes in the human brain: a stereological investigation. Neurobiol. Aging 18, 609615.

Tournier JD, Mori S, Leemans A, 2011. Diffusion tensor imaging and beyond. Magn. Reson. Med. 65, 1532-1556.

Vernooij, M.W., Ikram, M.A., Vrooman, H.A., Wielopolski, P.A., Krestin, G.P., Hofman, A., Niessen, W.J., Van der Lugt, A., Breteler, M.M.B., 2009. White matter microstructural integrity and cognitive function in a general elderly population. Arch. Gen. Psychiatry 66, 545-553.

Villain, N., Desgranges, B., Viader, F., de la Sayette, V., Mézenge, F., Landeau, B., Baron, J.C., Eustache, F., Chételat, G., 2008. Relationships between hippocampal atrophy, white matter disruption, and gray matter hypometabolism in Alzheimer's disease. J. Neurosci. 28, 6174-6181.

Villain, N., Fouquet, M., Baron, J.-C., Mézenge, F., Landeau, B., de la Sayette, V., Viader, F., Eustache, F., Desgranges, B., Chételat, G., 2010a. Sequential relationships between gray matter and white matter atrophy and brain metabolic abnormalities in early Alzheimer's disease. Brain 133, 3301-14.

Villain, N., Landeau, B., Groussard, M., Mevel, K., Fouquet, M., Dayan, J., Eustache, F., Desgranges, B., Chételat, G., 2010b. A Simple Way to Improve Anatomical Mapping of Functional Brain Imaging. J. Neuroimaging 20, 324-333.

Westlye, L.T., Walhovd, K.B., Dale, A.M., Bjørnerud, A., Due-Tønnessen, P., Engvig, A., Grydeland, H., Tamnes, C.K., Ostby, Y., Fjell, A.M., 2010. Life-span changes of the 
human brain white matter: diffusion tensor imaging (DTI) and volumetry. Cereb. Cortex 20, 2055-2068.

Wu, J.-T., Wu, H.-Z., Yan, C.-G., Chen, W.-X., Zhang, H.-Y., He, Y., Yang, H.-S., 2011. Aging-related changes in the default mode network and its anti-correlated networks: a resting-state fMRI study. Neurosci. Lett. 504, 62-67.

Zuendorf, G., Kerrouche, N., Herholz, K., Baron, J.-C., 2003. Efficient principal component analysis for multivariate 3D voxel-based mapping of brain functional imaging data sets as applied to FDG-PET and normal aging. Hum. Brain Mapp. 18, 13-21. 
Table 1: Demographic characteristics of the samples.

\begin{tabular}{cll}
\hline & CENTRE 1 & CENTRE 2 \\
\hline YOUNG & $\mathrm{N}=21$ & $\mathrm{~N}=19$ \\
Age & $28.7 \pm 5.5(21-37)$ & $25.5 \pm 4.1(22-37)$ \\
Education & $13.7 \pm 2.6(10-19)$ & $13.5 \pm 1.6(13-18)$ \\
ELDERLY & $\mathrm{N}=28$ & $\mathrm{~N}=29$ \\
Age & $69.9 \pm 6.5(60-84)$ & $69.2 \pm 8.2(60-85)$ \\
Education & $11.3 \pm 3.6(7-20)$ & $12.5 \pm 3.6(9-18)$ \\
\hline
\end{tabular}

Age and education are indicated in years as mean \pm standard deviation (minimum, maximum). 


\section{Figure captions}

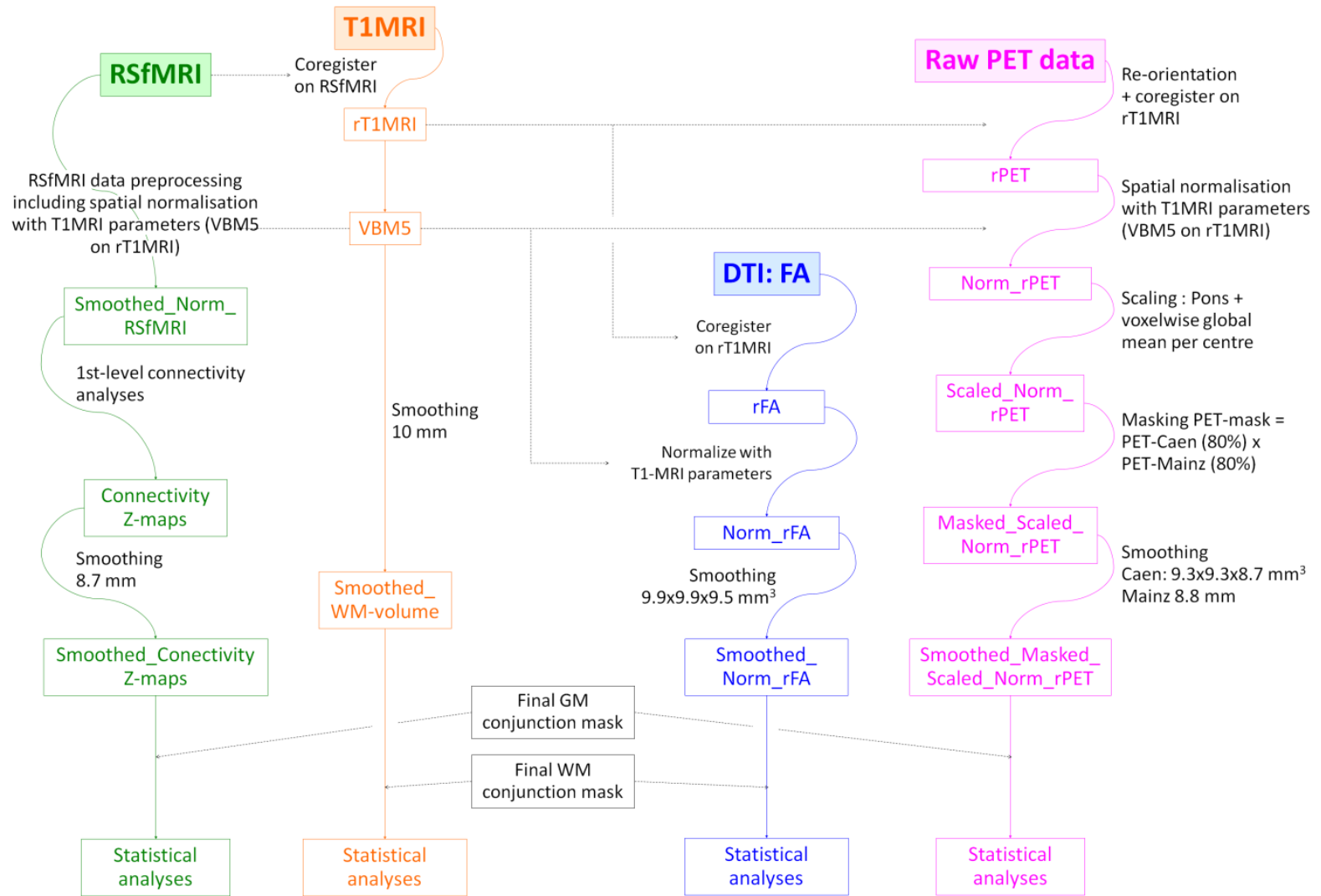

Figure 1: Preprocessing scheme of RSfMRI, WM volume from T1-weighted MRI (VBM),

DTI-FA and FDG-PET data (see text for details). 


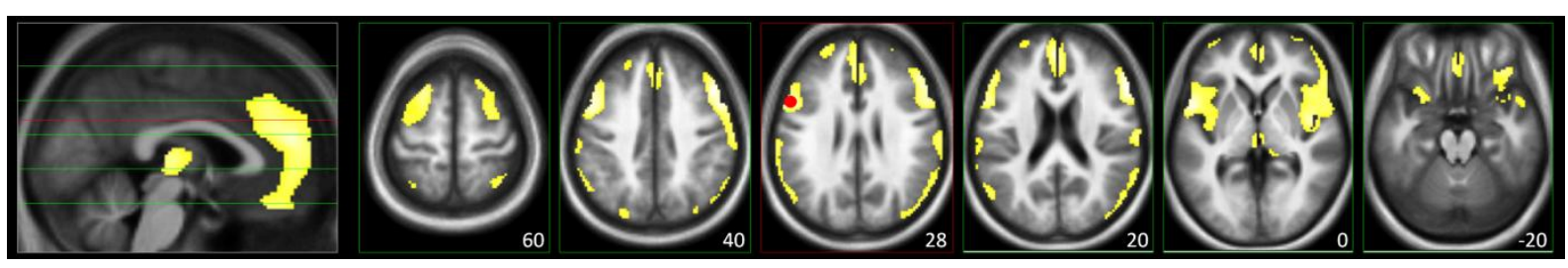

Figure 2: Results of the group comparison of FDG-PET data from Centre 1 and Centre 2 (conjunction analysis; elderly < young; $\mathrm{p}(\mathrm{FWE})<10^{-5}$ ) superimposed on axial slices (MNI z coordinate indicated on the bottom right of each slice). The LIFJ seed is indicated in red. 


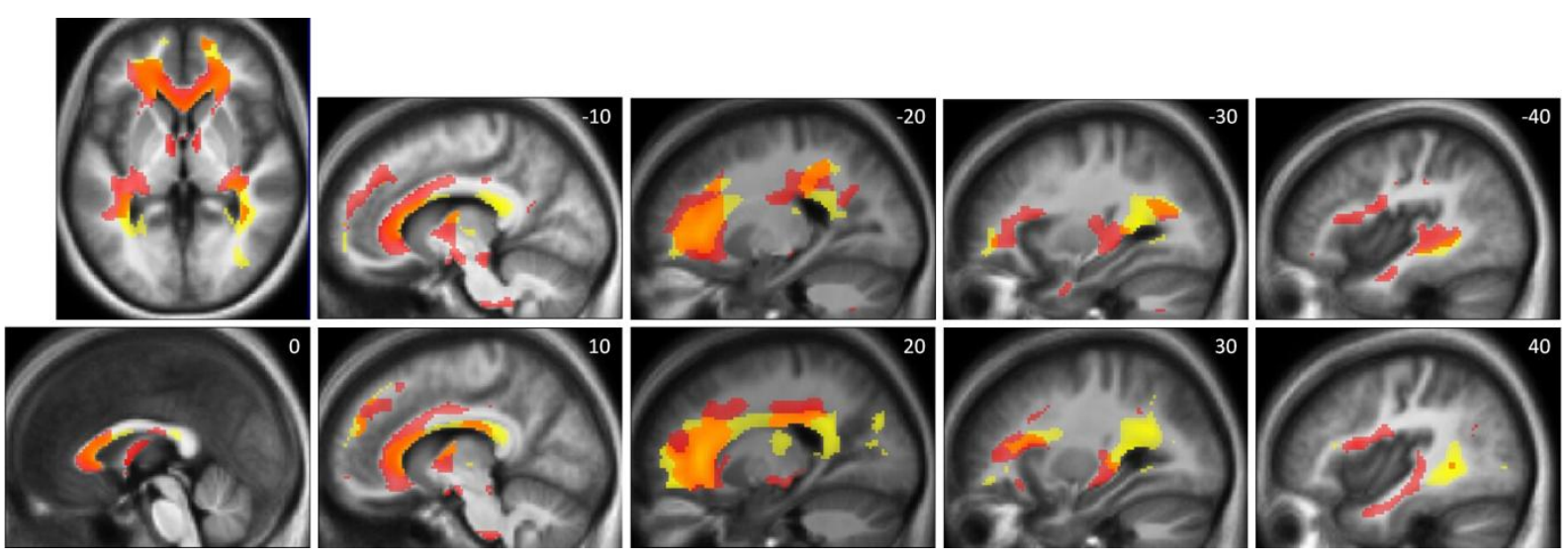

Figure 3: Results of the group comparisons (elderly < young) of WM volume data from Centre 1 (yellow; $\mathrm{p}(\mathrm{FDR})<0.05, \mathrm{k}>20)$ and DTI data from Centre 2 (red; $\mathrm{p}(\mathrm{FWE})<0.05$, $\mathrm{k}>20)$ superimposed on one axial slice $(\mathrm{MNI} z=0)$ and on sagittal slices (MNI x coordinate indicated on the top right of each slice).This figure illustrates the striking overlap between both profiles of WM alteration despite the fact that they were obtained using different techniques (VBM on T1-MRI versus FA with DTI) and two independent samples. The results were however more significant using DTI-FA as a more severe statistical threshold was used for DTI than for WM volume to obtain comparable extent of results. 


\section{A. LIFJ connectivity in young}
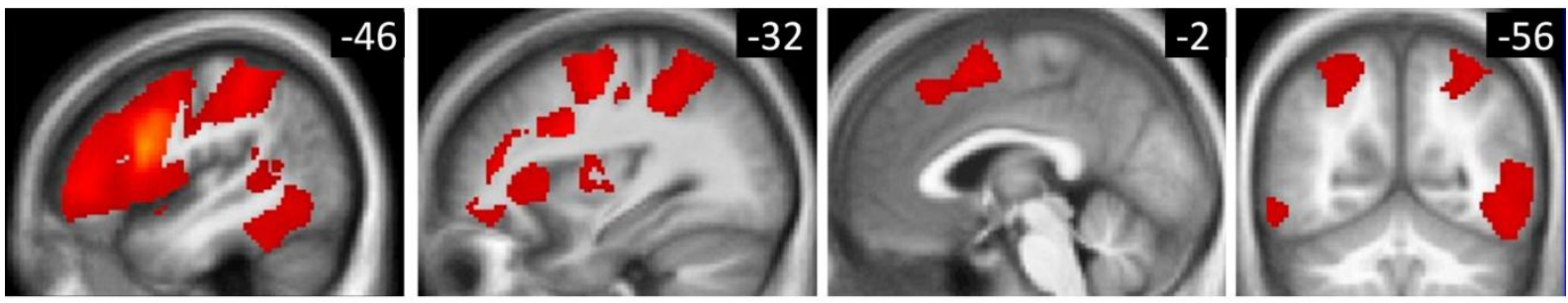

\section{B. LIFJ connectivity in young and elderly}
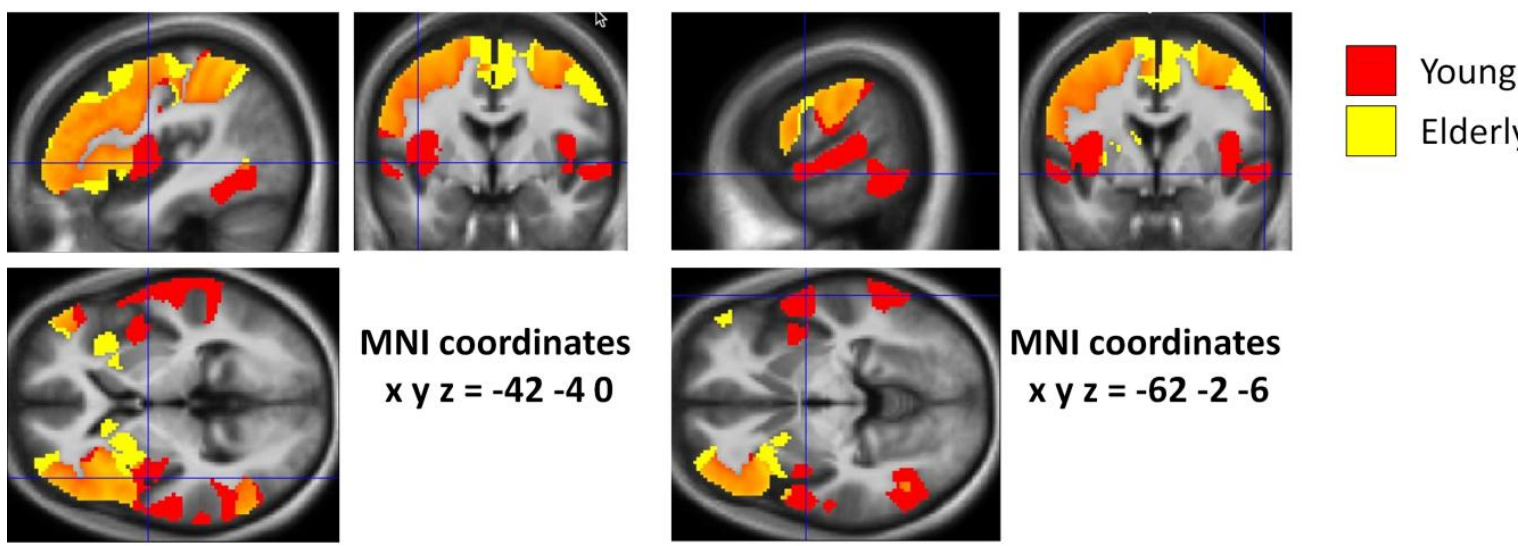

\section{Elderly}

MNI coordinates

$x$ y $z=-42-40$

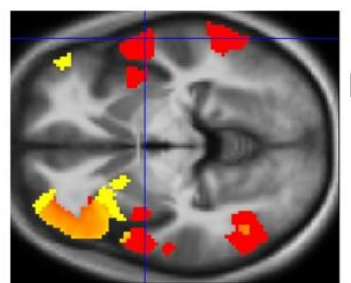

MNI coordinates

$x$ y $z=-62-2-6$

\section{LIFJ connectivity young > elderly}
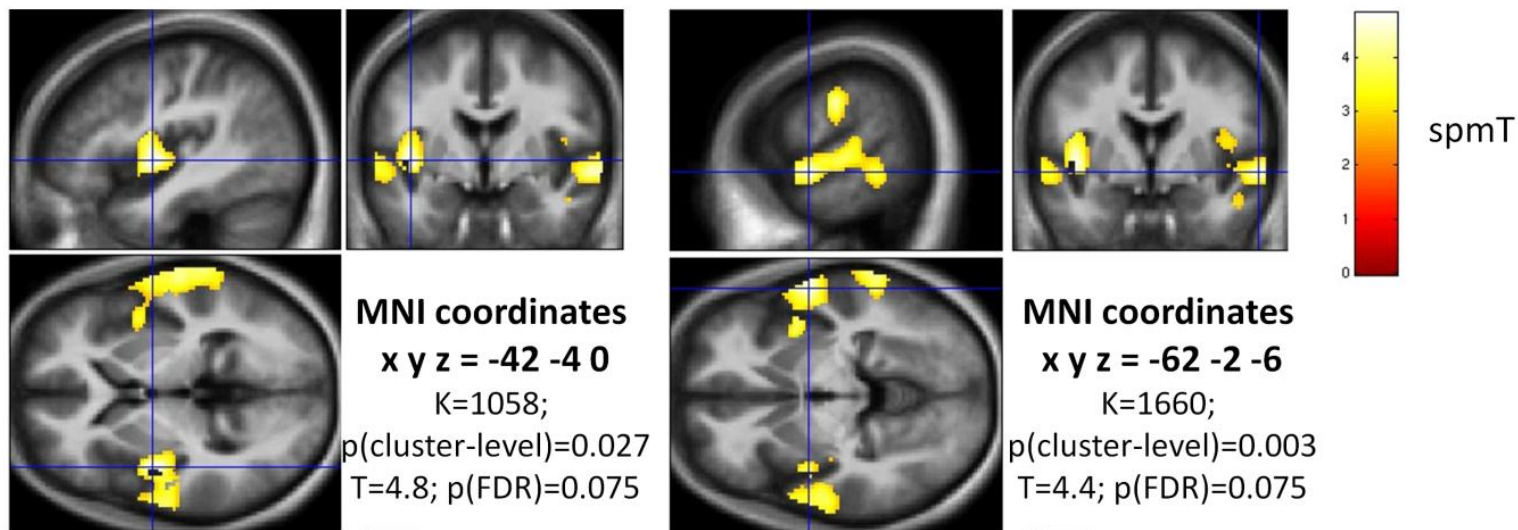

\section{MNI coordinates}

$x$ y $z=-42-40$

$\mathrm{K}=1058$;

$p($ cluster-level $)=0.027$

$\mathrm{T}=4.8 ; \mathrm{p}(\mathrm{FDR})=0.075$

\section{MNI coordinates}

$x$ y $z=-62-2-6$

$\mathrm{K}=1660$;

$p$ (cluster-level $)=0.003$

$\mathrm{T}=4.4 ; \mathrm{p}(\mathrm{FDR})=0.075$

Figure 4: Results of the resting-state fMRI LIFJ functional connectivity analyses. A: Results

of the one-sample analysis in the young participants from Centre $1(\mathrm{p}(\mathrm{FDR})<0.05 ; \mathrm{k}>20)$.

This figure illustrates the normal functional connectivity of the LIFJ, and the slices have been

selected for the sake of comparison with one previous study reporting the coactivations and functional connectivity of the LIFJ (Sundermann and Pfleiderer, 2012; Figure 1; x or y

coordinates indicated in the upper right of each slice). B: Results of the one-sample analysis in the young (red) and old (yellow) participants (both at $\mathrm{p}(\mathrm{FDR})<0.05 ; \mathrm{k}>20$ ) centred on the two most significant peaks where elderly showed lower connectivity compared to young 
subjects in the group comparison analysis for the sake of illustration. C: Results of the group comparison (elderly < young; $\mathrm{p}($ uncorrected $)<0.005, \mathrm{k}>50)$ centred on the two most significant peaks and their corresponding statistics. 

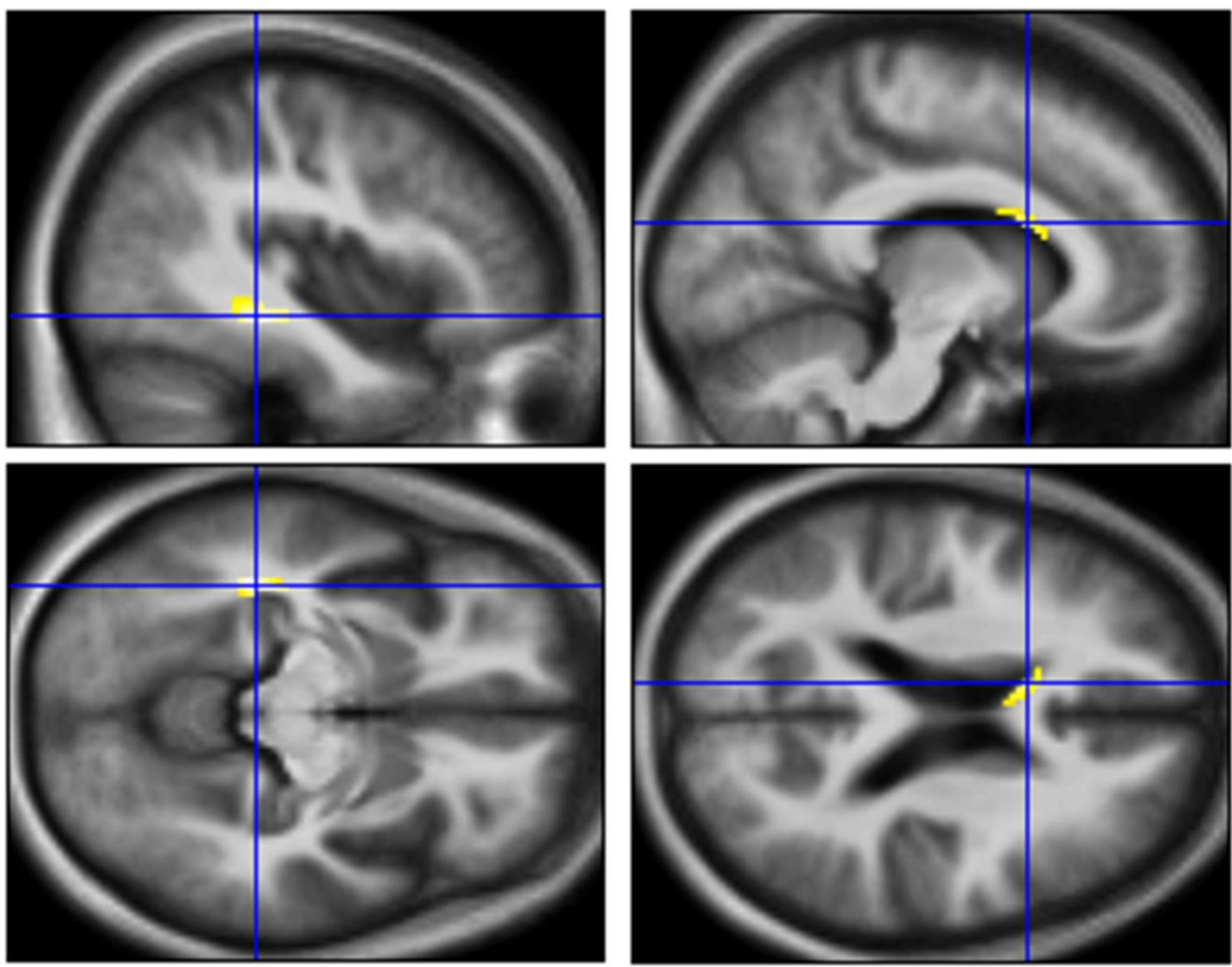

MNI coordinates

$$
\begin{gathered}
x y z=-\mathbf{4 0}-\mathbf{3 4}-\mathbf{1 0} \\
K=66 ; \\
T=4.6 ; p(F D R)=0.14 ; \\
p(F W E)=0.09
\end{gathered}
$$

\section{MNI coordinates}

$$
\begin{gathered}
\text { x y } z=\mathbf{- 1 0}-\mathbf{3 4} \mathbf{- 1 0} \\
\mathrm{K}=124 ; \\
\mathrm{T}=3.9 ; p(F D R)=0.14 \\
p(F W E)=0.34
\end{gathered}
$$

Figure 5: Results of the regression analysis between LIFJ metabolism and WM volume within the elderly (uncorrected $\mathrm{p}<0.005 ; \mathrm{k}>50$ ) superimposed on sagittal and axial slices centred on the two significant cluster peaks and their corresponding statistics. 


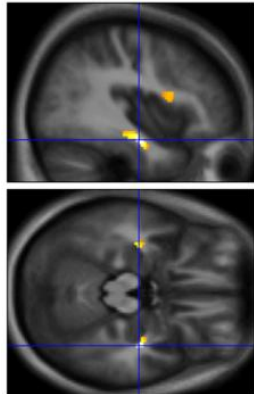

MNI coordinates $x y z=40-12-18$ $\mathrm{K}=67$;

$T=6.1 ; p(F D R)=0.007$

$p(F W E)=0.004$

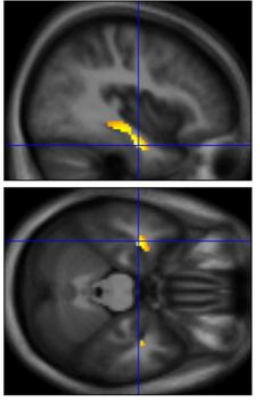

MNI coordinates $x y z=-38-10-24$ $\mathrm{K}=206$;

$\mathrm{T}=5.4 ; \mathrm{p}(\mathrm{FDR})=0.01$

$p(F W E)=0.02$

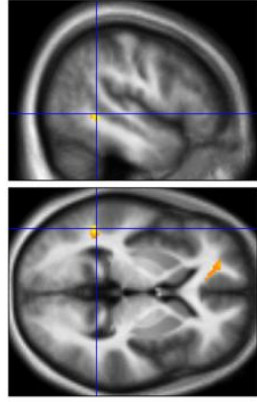

MNI coordinates $x y z=-48-460$ $\mathrm{K}=54$; $\mathrm{T}=4.2 ; \mathrm{p}(\mathrm{FDR})=0.03$ $p(F W E)=0.21$

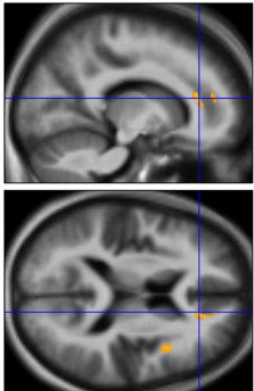

MNI coordinates $x y z=143614$ $\mathrm{K}=51$;

$\mathrm{T}=3.6 ; \mathrm{p}(\mathrm{FDR})=0.04$ $p(F W E)=0.55$

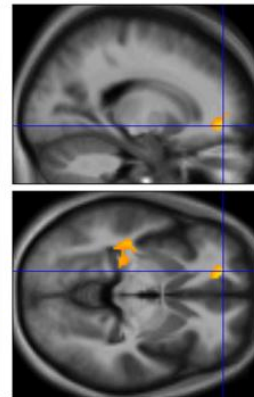

MNI coordinates $x y z=-1848-6$ $\mathrm{K}=101$;

$T=3.6 ; p(F D R)=0.04$ $p(F W E)=0.56$

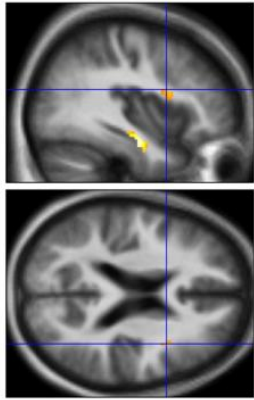

MNI coordinates $x y z=381020$ $\mathrm{K}=53$;

$T=3.5 ; p(F D R)=0.05$ $p(F W E)=0.65$

Figure 6: Results of the regression analysis between LIFJ metabolism and DTI-FA within the elderly (uncorrected $\mathrm{p}<0.005 ; \mathrm{k}>50$ ) superimposed on sagittal and axial slices centred on the six significant cluster peaks and their corresponding statistics. 


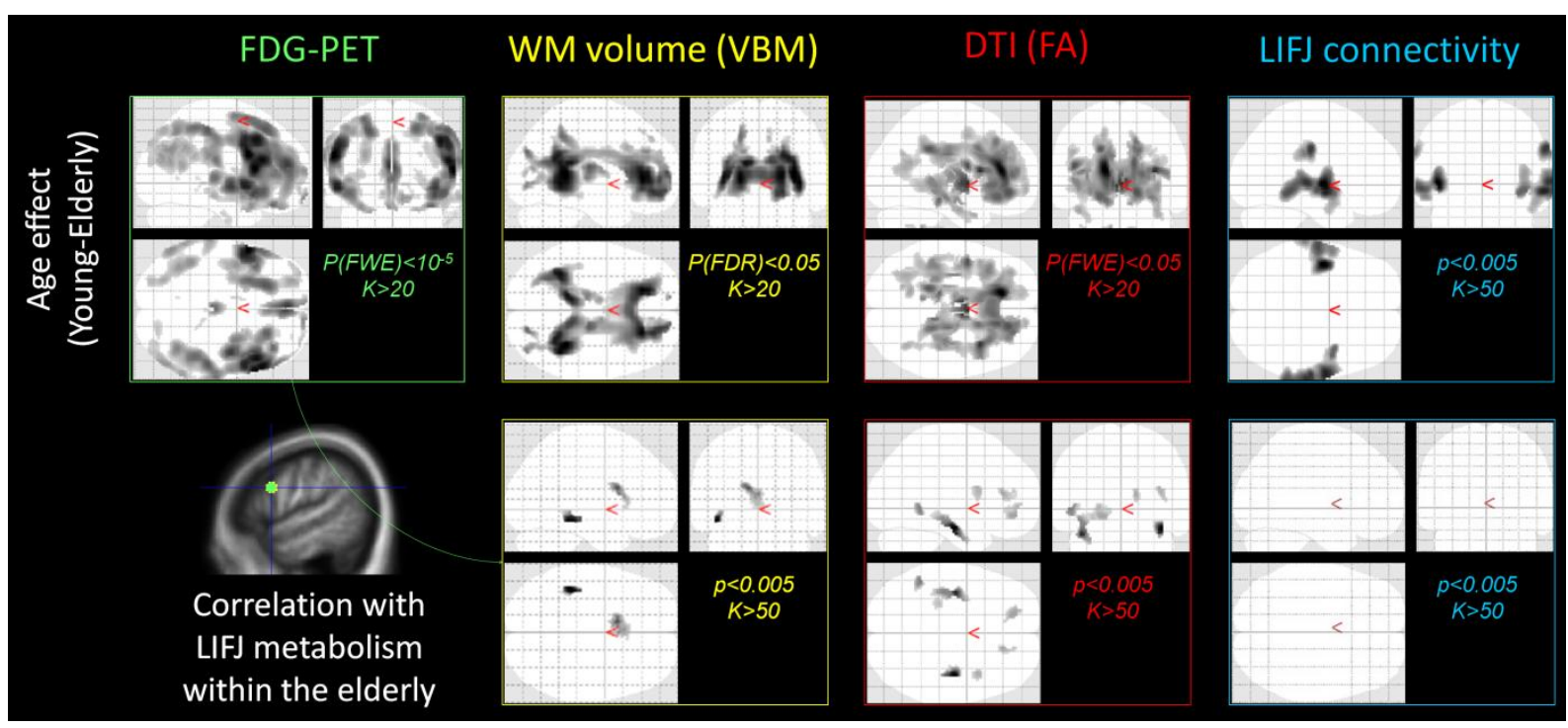

Figure 7: Sum-up of all the results of the present study including the group comparison

analyses on the four imaging modalities (top row) and the correlation analyses between LIFJ FDG-PET metabolism and the three other neuroimaging modalities within the elderly (down row). Age-related decrease of metabolism and alteration of WM integrity in elderly compared to young subjects overall predominated in the frontal lobe and were found to be massive for FDG-PET and DTI-FA, less but still important for WM volume, while LIFJ functional connectivity was only slightly modified. Age-related LIFJ metabolism decrease with age was found to correlate with WM macroscopic (WM volume) and microscopic (DTI-FA) integrity, especially in long association fronto-temporo-occipital fibers, but not to functional connectivity. 